\title{
MAS-PASTO: TALLER DE EXTENSIÓN DE PRÁCTICAS DE MANEJO SUSTENTABLE DEL PASTOREO EN CRÍA
}

\section{Mas-Pasto: Extension workshop on cattle breeding sustainable management}

\author{
Clavijo, M.P. ${ }^{1}$; Tufro, J. ; Cadaviz, N. ${ }^{1}$; Moreira, C.J. ${ }^{2}$; Vassallo, M.M. ; Kambo, S. ${ }^{3}$; \\ Miccoli, F. ${ }^{3}$; Grimoldi, A.A. ${ }^{1}$ \\ ${ }^{1}$ Catédra de Forrajicultura, \\ ${ }^{2}$ Cátedra de Extensión y Sociología Rurales y \\ ${ }^{3}$ Cátedra de Bovinos de Carne. Facultad de Agronomía, Universidad de Buenos Aires \\ mclavijo@agro.uba.ar
}

\section{RESUMEN}

Los productores ganaderos pastoriles de pequeña y mediana escala de la Provincia de Buenos Aires exhiben un bajo nivel de adopción de tecnologías de procesos y baja producción de carne. La difusión de estas tecnologías por parte de universidades e INTA es escasa. Dentro del proyecto de extensión MAS-PASTO dirigido por la cátedra de Forrajicultura de la FAUBA (cooperan Sociología y Extensión Agrarias y Bovinos de Carne), se desarrolló durante 2019 un taller a campo con el objetivo de construir un vínculo para el intercambio, la extensión y la transferencia de tecnología entre la FAUBA y productores ganaderos criadores de escala pequeña y mediana ( $\leq 300-1.000$ cabezas) que no reciben asistencia técnica sistemática. Se realizaron 5 jornadas a campo donde se abordaron de manera práctica y participativa las temáticas: características de las plantas forrajeras, siembra y utilización de pasturas y verdeos, ordenamiento y manejo del rodeo de cría, caracterización y mejoramiento de pastizales y el pastoreo rotativo como ejes del pastoreo sustentable. El taller se evaluó mediante encuestas, registros de campo y entrevistas; y se realizó un análisis de fortalezas, oportunidades, debilidades y amenazas del mismo. Se logró consolidar un método efectivo y replicable para la generación y transferencia de tecnologías de procesos desde la FAUBA hacia el sector productivo. Los participantes reforzaron sus conocimientos básicos y adquirieron competencias personales y profesionales para el diagnóstico y resolución de problemáticas a campo. El mejoramiento de los pastizales naturales surgió como área de sumo interés para enfocar líneas de trabajo de la FAUBA.

Palabras Clave: forrajes, cría vacuna, tecnología de procesos, extensión universitaria, sustentable.

\begin{abstract}
Small and medium-sized livestock farmers in BuenosAires evidence scarce implementation of process technology and low meat production. The diffusion of these technologies by universities and INTA is poor. As part of MAS-PASTO extension project managed by The FAUBA Forage Chair (Sociology and Rural Extension and Beef Cattle Chairs cooperated), during 2019 a field workshop was carried out with the aim of fostering communication as regards exchanges, the extension and technological findings between FAUBA and small and medium-sized livestock farmers ( $\leq 300-1,000$ head) that do not receive systematic technical assistance. During five working days, different topics such as: the characteristics of forage plants, sowing and pastures and forage annual crops usage, management of herd breeding, the characterization and improvement of natural grasslands and rotational grazing as base of sustainable grazing were developed in a practical and cooperative way. The workshop was evaluated through polls, field records and interviews; finally SWOT analysis was carried out. An effective and repeatable method for generating and transferring technological processes from FAUBA to the productive sector was consolidated. Participants reinforced their basic knowledge and acquired personal and professional skills for diagnosing and solving problems in the field. The improvement of natural grasslands was identified as an area of great interest to focus FAUBA lines of work.
\end{abstract}

Key words: for age, livestock breeding, process technology, Univer sity Extension, substainable.

Recibido: 17/jun/2020. Aceptado: 14/jul/2020 


\section{INTRODUCCIÓN}

Al igual que a nivel nacional, la producción ganadera de la Provincia de Buenos Aires exhibe una marcada brecha en la producción de carne entre establecimientos que reciben asistencia técnica profesional y aquellos que no la reciben (Pacín y Oesterheld, 2015). Esto se explica por el nivel de adopción tecnológica de los productores en cada caso. Feldkamp y Del Río (2011) estimaron que, si los productores con bajos niveles de adopción de tecnología pasaran a un nivel intermedio de adopción de tecnología, la producción nacional de carne podría mejorar como mínimo un $15 \%$. Los cambios entre niveles tecnológicos no involucran grandes modificaciones ni técnicas de avanzada sino que, en general, se refieren a tecnologías de procesos asociadas al manejo sustentable del pastoreo y ordenamiento del rodeo. A su vez, para los productores que no tienen asistencia técnica profesional, no hay espacios de formación como las escuelas de pastoreo que existen en departamentos de extensión de muchas universidades extranjeras.

En Argentina, la mayoría de las capacitaciones que ofrecen las universidades vinculadas a la producción ganadera son para profesionales. Existen otras instituciones públicas como INTA que tienen una oferta más variada de enseñanza (tanto para productores como encargados de campo), pero en todos los casos la difusión de tecnologías de procesos para la cría parece ser escasa. De la matrícula de Ingeniería Agronómica de la UBA, una amplia proporción de estudiantes quisieran dedicarse a actividades privadas que tienen implícitas la transferencia y gestión de conocimiento en las empresas agropecuarias y para ellos resulta de mucho interés participar de actividades de vinculación con el sector productivo durante su proceso de formación profesional. En este contexto, dentro del proyecto de extensión MAS-PASTO dirigido por la Cátedra de Forrajicultura de la Facultad de Agronomía de la UBA (FAUBA), (en cooperación con las Cátedras de Extensión y Sociología Rurales y Bovinos de Carne), se desarrolló durante 2019 un taller a campo con el objetivo de construir un vínculo para el intercambio, la extensión y la transferencia de tecnología entre la FAUBA y productores ganaderos criadores de escala pequeña ( $\leq 300$ cabezas) a intermedia ( $\leq 1.000$ cabezas) que no reciben asistencia técnica sistemática. Específicamente, el taller estuvo destinado a: 1) consolidar un método efectivo y replicable para la generación y transferencia de tecnologías de procesos desde la Facultad de Agronomía de la UBA hacia el sector productivo; 2) que estudiantes avanzados reforzaran sus conocimientos básicos y a la vez adquirieran competencias personales y profesionales para el diagnóstico y resolución de problemáticas a campo, y por ultimo; 3) que del intercambio entre el sector productivo, estudiantes y docentes surgieran nuevas líneas de trabajo para las cátedras de Forrajicultura y Bovinos de carne, vinculadas con las problemáticas detectadas.

\section{METODOLOGÍA}

El taller tuvo como actividad principal la realización de 5 jornadas de 1 día cada una (entre los meses de abril y agosto de 2019) en campos de productores ganaderos criadores pastoriles de la zona este de la provincia de Buenos Aires, para abordar in situ, de manera práctica y participativa, temáticas y problemáticas forrajeras y ganaderas, básicas para el manejo sustentable del pastoreo. Los 5 ejes temáticos del taller (Fig. 1) fueron: en primer lugar, la planta forrajera, donde se incluyeron las características generales de gramíneas y leguminosas forrajeras (tanto especies nativas del pastizal como especies exóticas que se siembran en pasturas y verdeos) en relación con su dinámica de crecimiento y su capacidad de rebrote post-pastoreo. En segundo y tercer lugar, los procesos productivos y de manejo más importantes de los principales recursos de los que forman parte las plantas forrajeras: las pasturas, verdeos y los pastizales. En el caso de pasturas y verdeos se enfocó en el proceso de siembra y la importancia del primer aprovechamiento una vez establecidos y en el caso de los pastizales, el foco estuvo puesto en los ambientes y comunidades que los configuran y posibilidades de mejoramiento. Estos tres ejes temáticos fueron considerados básicos y se combinaron con un cuarto eje que se concentró en el rodeo de cría, haciendo hincapié en la importancia su ordenamiento y manejo para poder acoplar sus requerimientos a los recursos forrajeros en un esquema de sustentabilidad. En el quinto eje, que integró los anteriores, se trabajó la propuesta integral de manejo sustentable del pastoreo. La propuesta consistió en el manejo de los forrajes bajo pastoreo rotativo, utilizando metodologías para estimar stock de forraje disponible en las parcelas de pastoreo, demostrando los indicadores visuales de entrada y salida de las parcelas y trabajando los criterios para definir tiempo de ocupación y descanso de las mismas.

Participaron 25 productores de cría pequeños y medianos (hasta 600 cabezas). Las actividades de las jorna 
das fueron planeadas de modo que cada jornada se realizó en un campo cuya problemática fuera afín a alguno de los 5 ejes temáticos. Para cada eje temático los docentes (6) realizaron el abordaje del marco teórico general en un gran grupo, y la puesta en práctica en pequeños grupos a través de estaciones de trabajo con la colaboración de un equipo de estudiantes avanzados de la carrera de agronomía (6). Los docentes fueron los responsables de la planificación, exposición de los temas y facilitación de las actividades en pequeños grupos a campo, mientras que los estudiantes además de colaborar en estas 3 actividades, registraron demandas y necesidades permanentemente y se focalizaron en la aplicación de sus conocimientos y habilidades previas.
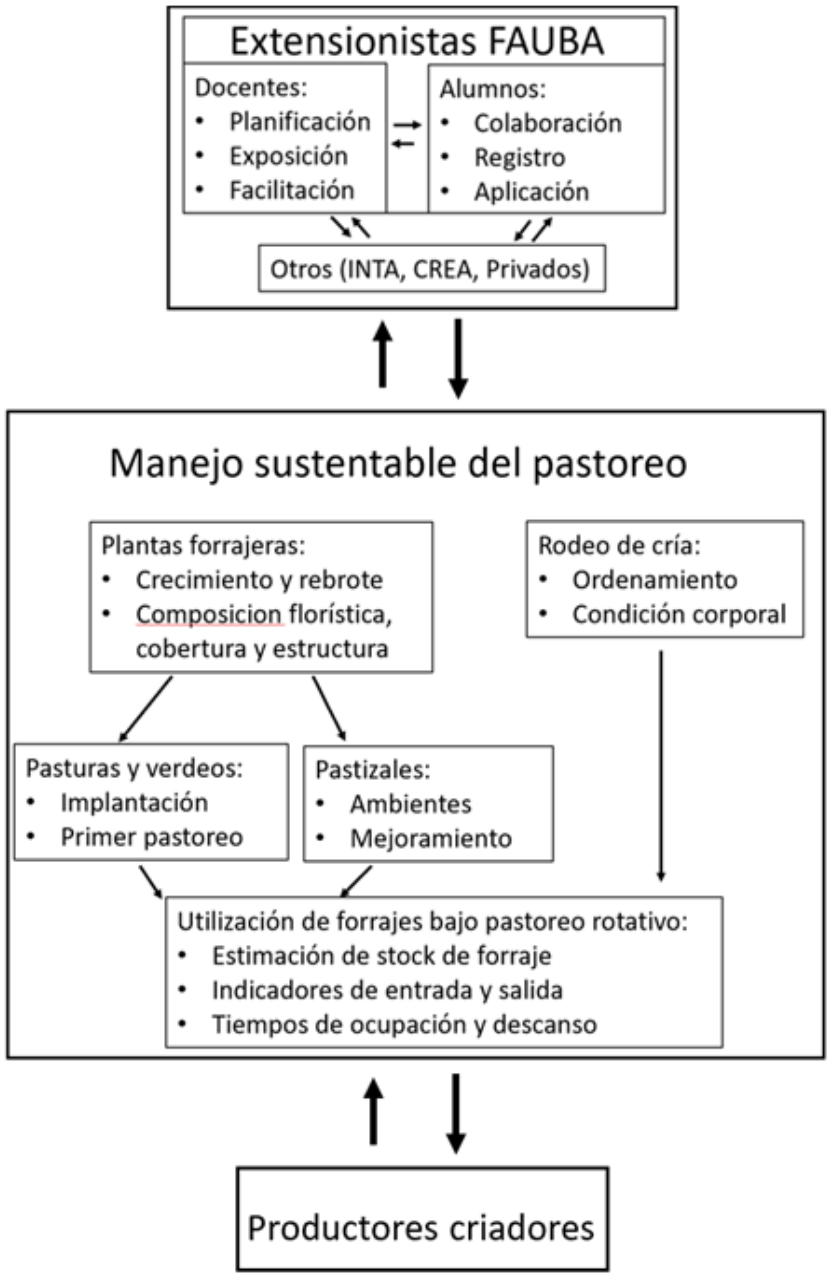

Figura 1. Ejes temáticos y procesos de transferencia de conocimiento del equipo de extensionistas hacia los productores.

La planificación del taller implicó viajes previos de docentes y estudiantes a los campos para definir al menos 3 estaciones de trabajo en cada uno. El armado de las actividades en dichas estaciones (reconocimiento de plantas, estimaciones de biomasa, mediciones de logro en implantación, diseño de circuitos de pastoreo, reconocimiento de comunidades de pastizal y estimación de condición corporal del rodeo de cría y reconocimiento de indicadores para determinar entrada y salida de las parcelas bajo pastoreo rotativo). Se realizaron trabajos previos de laboratorio (estimaciones de materia seca, calibraciones de herramientas, identificación taxonómica de especies) así como también talleres de discusión con profesionales de INTA y privados (semilleros, grupos CREA) que realizan actividades de extensión sobre los mismos temas. Para la exposición del marco teórico en cada jornada se elaboró material de apoyo impreso e individual con el resumen de los conceptos tratados y las consignas de las actividades de campo. Cada una de las jornadas de campo, y el taller en general, fueron evaluados mediante encuestas impresas o formularios de Google entregados a los productores y 
estudiantes, registros escritos de los estudiantes sobre demandas de los participantes y entrevistas individuales a los productores los cuales fueron analizadas por uno de los estudiantes junto con la realización de un análisis de Fortalezas, Debilidades, Oportunidades y Amenazas (FODA) como trabajo preprofesional para acceder al título de Ingeniero Agrónomo.

La información cualitativa y cuantitativa recopilada se sistematizó e interpretó siguiendo la metodología propuesta por Huberman y Miles (1994) en tres fases, la reducción a grupos o categorías, la exposición en formas visuales como diagramas o cuadros y las conclusiones, que se sacaron a partir de la interpretación de los datos expuestos (Coffey y Atkinson, 2003). La codificación de la información cualitativa, se concentró en las múltiples palabras que los asistentes usaron para referirse a los ejes temáticos, el material impreso, las actividades de campo y la participación, usando estos tópicos como categorías generales. Las funciones de esta codificación fueron las de clasificar y ordenar la información con el criterio de favorecer la interpretación de los datos, teniendo como índices de referencia para agrupar la información las percepciones que transmitían las valoraciones de los encuestados. Una vez organizados de este modo, los datos fueron reorganizados en subcategorías de respuestas negativas, positivas, o muy positivas. El desarrollo de las subcategorías no fue profuso, de modo de no acotar y fragmentar demasiado los datos, evitando la pérdida de información contextual potencialmente valiosa por una diferenciación excesiva de fragmentos de relatos. El cruce de información cualitativa y cuantitativa dentro de una misma encuesta, y entre encuestas, suscitó una instancia de resignificación de las interpretaciones que generaba el análisis por separado de cada tipo de información y las frecuencias de respuesta negativas, positivas, o muy positivas para cada pregunta.

El análisis de los testimonios y consideraciones de asistentes y estudiantes favoreció el desarrollo de un proceso de interpretación del taller sustentado en datos emergentes del contacto e interacción directa de los docentes con ambos grupos. Esto permitió valorar entre los resultados del taller el proceso de construcción de nuevos saberes, y reconstrucción de conceptualizaciones respecto a saberes que los asistentes portaban previamente (Hernández Sampieri et al, 2006).

Con toda la información recopilada se realizó un análisis de Fortalezas, Debilidades, Oportunidades y Amenazas (FODA), teniendo en cuenta los aportes de López Cañizares en esta materia (López Cañizares, 2019). La información que se tomó como insumo para el análisis FODA incluyó la totalidad de los datos generados, procesados y sistematizados de las encuestas realizadas por los productores y los estudiantes. Entre las fortalezas se consideraron las características que el taller no debería cambiar porque se evalúan positivamente. Para el caso de las debilidades se contemplaron los puntos que se pueden mejorar, y que están al alcance de los organizadores. Se identificó como situación externa, asociada a oportunidades y amenazas, lo surgido de los puntos en los que no se puede lograr un cambio o una intervención directa, por no depender del equipo que organiza el taller. Esto permite lograr una mayor comprensión del ámbito en el que se desarrolla el taller, con sus restricciones y potencialidades a corto, mediano y largo plazo. Para la identificación de oportunidades se aglutinaron aquellos ítems ajenos al taller que este puede capitalizar, como por ejemplo la falta de presencia de instituciones abocadas a la transferencia de tecnologías en materia ganadera en la zona de intervención. En el caso de las amenazas, se condensaron los aspectos que pueden perjudicar los objetivos o la consolidación del taller, siendo que su resolución no está al alcance de los organizadores del mismo.

\section{RESULTADOS OBTENIDOS}

\section{Participación y recepción de la propuesta de extensión}

Los productores recibieron la propuesta con entusiasmo y participaron activamente durante todas las jornadas. Cuando se los consultó sobre su valoración de los ejes temáticos de cada jornada, el material de apoyo impreso, las actividades a campo en pequeños grupos y la participación tanto en la discusión del marco teórico como en las actividades en pequeños grupos a campo, las respuestas fueron siempre positivas siendo el contenido y las actividades realizadas los aspectos mejor evaluados (Fig. 2). 


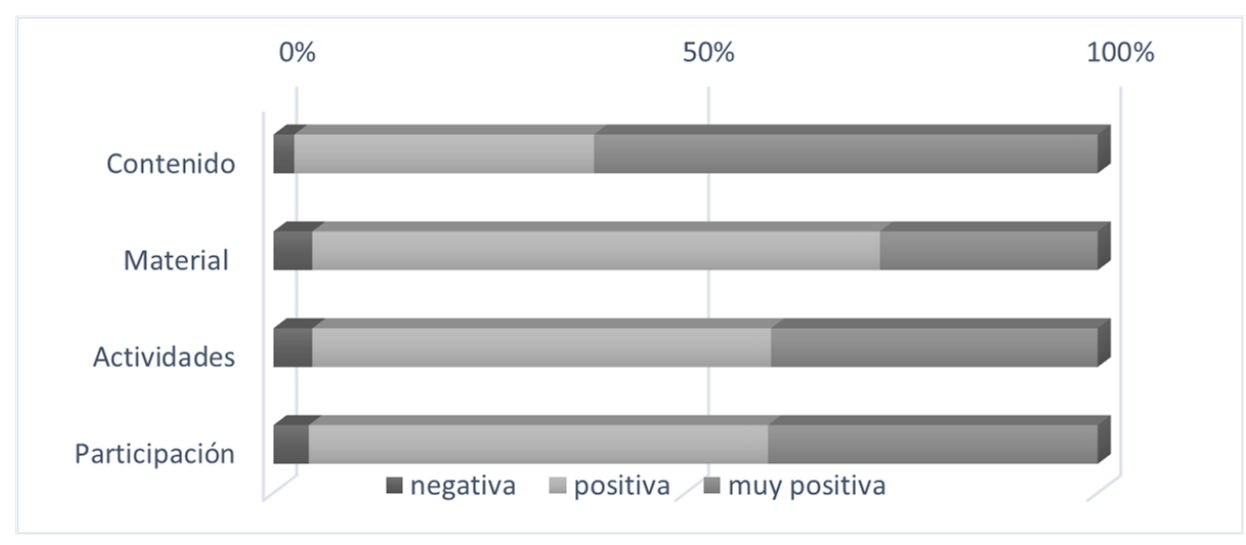

Figura 2. Síntesis de la valoración de las jornadas por parte de los productores, realizada mediante palabras descriptoras reducidas a las categorías negativa, positiva y muy positiva (en \% del total de respuestas) según la metodología de Huberman y Miles (1994). Se evaluó eje temático de cada jornada (utilidad, claridad y tiempo de exposición), material de apoyo impreso (diseño, extensión y lenguaje), actividades a campo en pequeños grupos (cantidad, calidad y tiempos) y participación tanto en la discusión del marco teórico como en las actividades a campo en pequeños grupos (facilitación en la discusión y facilitación en las actividades).

\section{Productos de la intervención}

Sobre la mayoría de los temas tratados en el taller los productores declararon en las encuestas que tenían un nivel de conocimiento intermedio, y que luego del taller el aumento de conocimiento sobre los mismos temas fue alto, en el $70 \%$ de los casos; y medio, en más del $20 \%$ (Fig. 3).

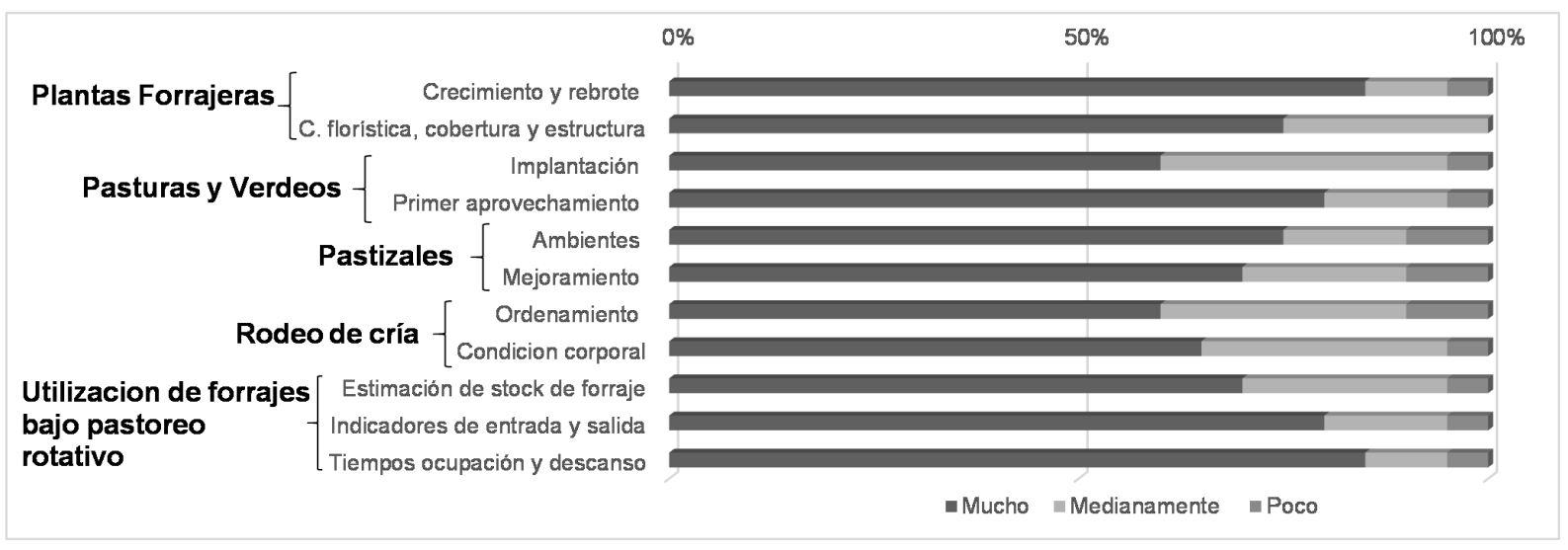

Figura 3. Percepción de los productor es sobre el aumento de conocimiento sobre los contenidos desarrollados dentro de los 5 ejes temáticos (se expresa en \% del total de respuestas).

Los productores identificaron a la producción y el aprovechamiento del forraje como los temas que mayor impacto generaban en sus sistemas pastoriles. Por otro lado, del registro realizado por los estudiantes en las actividades de campo y en las entrevistas surgió como un tema de mucho interés la generación de propuestas de mejoramiento de los pastizales naturales. El $80 \%$ de estos productores respondieron que en caso de aplicar las herramientas del taller obtendrían mejoras significativas en la producción de sus sistemas. En su mayoría, mencionaron que no habría motivos que impidan poner en práctica en forma inmediata las tecnologías desarrolladas durante el taller.

En el caso de los estudiantes, los conocimientos previos al taller fueron los correspondientes a la carrera de grado de agronomía, en todos los casos con la totalidad de las materias aprobadas. Ellos percibieron que las exposiciones del marco global teórico y las actividades en pequeños grupos a campo, así como los intercambios con los docentes durante su colaboración en el armado del taller, y la posibilidad de discusión de los mismos 
temas con extensionistas de otras instituciones; les permitieron reforzar los conocimientos previos y adquirir otros nuevos sobre los 5 ejes temáticos del taller. Los estudiantes consideraron que el eje temático que relativamente les aportó menor grado de nuevos conocimientos fue el del ordenamiento y manejo del rodeo de cría, que al ser conocimientos básicos son vistos ampliamente en las producciones animales de la carrera de agronomía. Respecto al desarrollo de su competencia como extensionistas, informaron que la habilidad que les resultó más dificultosa fue la de exponer contenidos teóricos en la presentación de los temas, y luego la coordinación de las actividades en pequeños grupos a campo (Fig. 4 a y b).

a) Conocimientos adquiridos

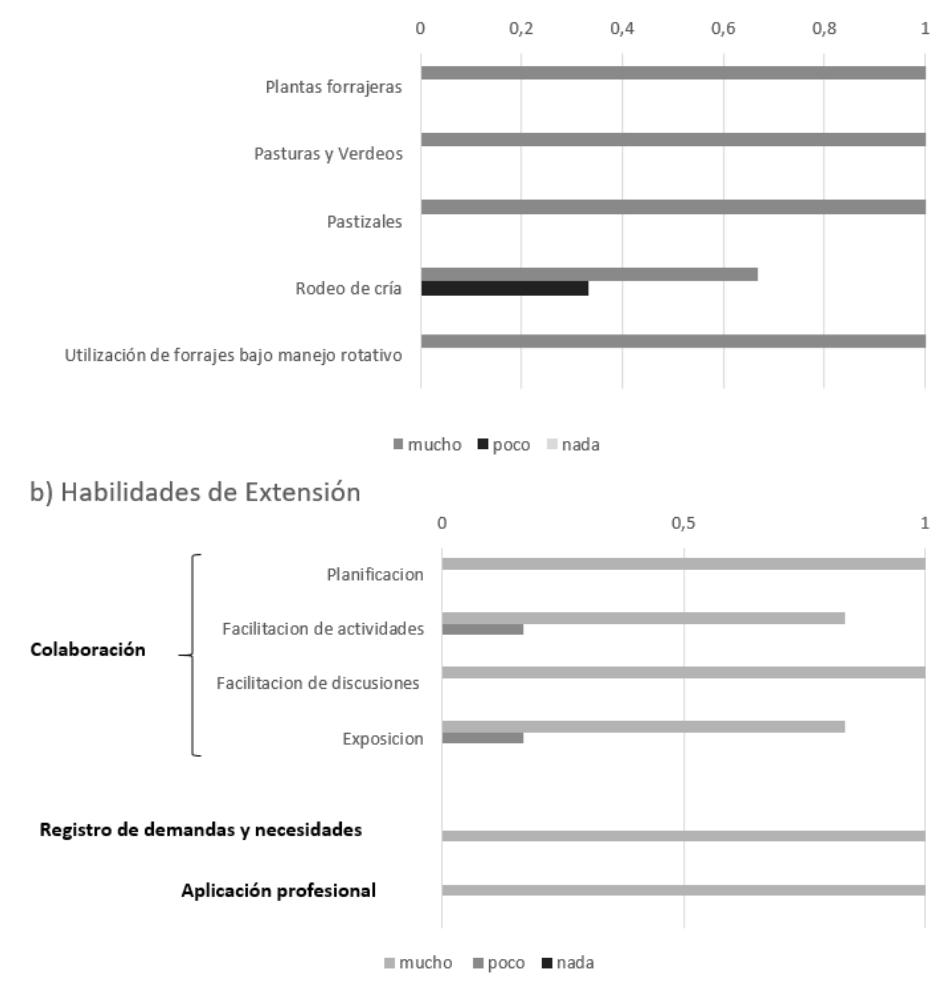

Figura 4. Percepción de los estudiantes sobre: a) su aumento de conocimiento por eje temático y b) sobre su adquisición de habilidades de extensión (se expresa en \% de las respuestas).

En todos los casos los estudiantes expresaron que las expectativas que tenían al sumarse al proyecto se cumplieron en un $100 \%$. Estas fueron: afianzar conocimientos, conocer sistemas productivos reales, nutrirse del intercambio con docentes y productores, brindar un servicio a la comunidad, y tomar contacto con el sector productivo.

\section{Análisis de Fortalezas, Debilidades, Oportunidades y Amenazas (FODA)}

Entre las fortalezas del taller se rescató la gran interacción en los grupos de trabajo de docentes, estudiantes y productores; y el diseño de las jornadas (tiempos, actividades prácticas realizadas y lenguaje utilizado). Como principales debilidades se observó que el material de apoyo entregado en algunos casos les resultó escaso a los asistentes; el rol de los estudiantes no fue suficientemente especificado a los productores; y que los grupos de trabajo no se armaron en función de los niveles previos de conocimiento de sus integrantes, razón por la cual algunas actividades como la realización de cálculos (por ejemplo: producción de carne por hectárea y por año) en algunos casos resultaron dificultosas. Como oportunidades, destacamos el interés del sector productivo ya que la propuesta rápidamente completó su cupo y mantuvo muy alto nivel de asistencia a las jornadas y participación en las mismas; y la demanda de estudiantes para formarse en la actividad de extensión. Finalmente, entre las amenazas para la consolidación y efectividad de la propuesta, señalamos el elevado costo de financiamiento del taller y la falta de certeza sobre la futura aplicación de las tecnologías desarrolladas en los sistemas de producción propios de los asistentes. 


\section{CONSIDERACIONES FINALES}

En base a la experiencia del taller sobre prácticas de manejo sustentable del pastoreo en cría, concluimos que la propuesta de extensión logró contemplar no sólo la transferencia de saberes en tecnologías de procesos, sino también la posibilidad de discutirlos y practicarlos a campo. El taller fue muy aceptado por los productores de cría pequeños y medianos, que no tienen asistencia técnica permanente, lo cual apoya la idea de replicar el taller en próximas ediciones. Habiéndonos planteado como primer objetivo del proyecto consolidar un método efectivo y replicable para la generación y transferencia de tecnologías de procesos desde la FAUBA hacia el sector productivo, creemos que, si bien el taller realizado es susceptible de mejoras en los aspectos que se identificaron como sus debilidades, el objetivo fue alcanzado. Las encuestas realizadas revelaron que tanto la interacción en los grupos de trabajo de docentes, estudiantes y productores, así como el diseño de las jornadas (tiempos, actividades prácticas realizadas y lenguaje utilizado) les permitieron a los asistentes entender e incorporar los contenidos de los ejes temáticos con la voluntad de llevarlos a la práctica en sus propios establecimientos.

Con respecto al propósito de que un grupo de estudiantes avanzados reforzaran sus conocimientos básicos y a la vez adquirieran competencias personales y profesionales para el diagnóstico y resolución de problemáticas a campo concluimos que, tanto el bagaje de conocimientos traídos por ellos de la carrera de grado, como su expectativa personal, les permitió capitalizar todas las acciones del taller. Ellos manifestaron que la exposición y la facilitación de actividades fueron las habilidades que menos incorporaron ya que estas acciones eran llevadas a cabo mayormente por el equipo docente, tal vez, sin darles el espacio y la confianza suficiente para que las realicen ellos. Consideramos que a futuro deberíamos pautar un rol aún más activo de los estudiantes en todas las instancias del taller.

Finalmente, si bien en la mayoría de los temas los productores manifestaron haber incorporado muchos conocimientos, la información de registros y entrevistas realizadas por estudiantes a lo largo de las jornadas nos orienta hacia la generación de propuestas de mejoramiento de los pastizales naturales como área de sumo interés para enfocar líneas de trabajo de la FAUBA.

\section{BIBLIOGRAFÍA}

Coffey, A. y Atkinson, P. (2003) "Variedad de datos y variedad de análisis" y "Los conceptos y la codificación”. En Coffey, A. y P. Atkinson (Autores): Encontrar el sentido a los datos cualitativos. Estrategias complementarias de investigación. Universidad Nacional de Antioquia, Colombia, pp. 1-63.

Feldkamp, C. y Del Rio, J. (2011). Cría Vacuna. 2015. Ciudad Autónoma de Buenos Aires: Asociación Argentina de Consorcios Regionales de Experimentación Agrícola.

Hernández Sampieri, R.; Fernández, C.; y Pilar Baptista, L. (2006) Metodología de la investigación. $4^{\circ}$ Edición. McGraw-Hill, México, pp. 2-31.

Huberman, M. y Miles, M. (1994) "Manejo de datos y métodos de análisis", en Denzin, N. y Lincoln, Y. (eds.) Handbook of Qualitative Research, Londres, Sage

López Cañizares, D.B. (2019) "Uso de metodologías para el análisis y diagnóstico de sistemas de producción agropecuarios”.Ciencia, Innovación, Tecnología, Ambiente y Sociedad, Vol. 1, ํㅜ 2, Universidad Santo Tomás, Barranquilla, Colombia, pp. 21-27.

Pacin, F. \& Oesterheld, M. (2015) Closing the technological gap of animal and crop production through technical assistance. Agricultural Systems 137: pp. 101-107. 